\title{
Behavioral Factors Associated with Under-Five Diarrhoeal Morbidity in Rural Community of Gummer Woreda, Gurage Zone, Southern Ethiopia: Community Based Cross-Sectional Study
}

\author{
Eyasu Gambura1, Wondwossen Birke², Gudina Terefe², Tadele Yohanes³, Tariku Laelago3,4* \\ ${ }^{1}$ Woreda Health Office, Areka, Ethiopia \\ ${ }^{2}$ Department of Environmental Health, Jimma University, Jimma, Ethiopia \\ ${ }^{3}$ Department of Health Extension, Hossana College of Health Sciences, Hossana, Ethiopia \\ ${ }^{4}$ Department of Health Information Technician, Hossana College of Health Sciences, Hossana, Ethiopia \\ Email: eyasugabura@yahoo.com,wondbrk@aol.com, guditerefe@gmail.com, tadejoha@gmail.com, \\ *tarikulalago@gmail.com
}

Received 27 March 2016; accepted 10 April 2016; published 13 April 2016

Copyright (C) 2016 by authors and OALib.

This work is licensed under the Creative Commons Attribution International License (CC BY). http://creativecommons.org/licenses/by/4.0/

(c) (i) Open Access

\section{Abstract}

Background: Diarrheal diseases are the second leading cause of child deaths globally and now considered to be the leading cause of child deaths in Africa. In Ethiopia, diarrheal diseases are also important child health problems. This study aimed to investigate the effect of behavioral factors on the under-five diarrheal morbidity in the rural communities of Gummer Woreda, Southern Ethiopia. Methods: Community-based cross-sectional study was conducted from April 30/2012 to June 4/2012 in the rural community of Gummer Woreda, Southern Ethiopia. A total of 634 households that had at least one under-five child were randomly selected from the nine Kebeles and included in the study. Structured pre-tested questionnaire and observation checklist were applied to collect data. Bivariate and multivariate logistic regressions were used to test associations between outcome and explanatory variables. Odds ratios at $95 \% \mathrm{CI}$ were computed to measure the strength of the association between the outcome and the independent variables. P-value $<0.05$ was considered as a statistically significant in multivariate analysis. Result: Out of 634 households with at least one under five child, $611(96.4 \%)$ gave informed consent and were included in the study. Respondents who used bottle feeding method (AOR: $2.866,95 \% \mathrm{CI}$ : $(1.588,5.170)$, practice of hand washing after visiting latrine (AOR: $2.080,95 \% \mathrm{CI}$ : $(1.076,4.021)$, the presence of observable faeces around the pit hole/slab in their latrine (AOR: 2.391, 95\%CI: $(1.444,3.959)$, children from those mothers who had history of diarrhea (AOR: 3.158, 95\%CI: $(1.240,8.045)$ were significantly associated with childhood diarrhea. With regard to diarrheal morbidity prevalence, the two-week

\footnotetext{
${ }^{*}$ Corresponding author.
}

How to cite this paper: Gambura, E., Birke, W., Terefe, G., Yohanes, T. and Laelago, T. (2016) Behavioral Factors Associated with Under-Five Diarrhoeal Morbidity in Rural Community of Gummer Woreda, Gurage Zone, Southern Ethiopia: Community Based Cross-Sectional Study. Open Access Library Journal, 3: e2570. http://dx.doi.org/10.4236/oalib.1102570 
period prevalence prior to the survey was $14.6 \%$. Conclusion: Maternal child care and hygienic behavioral practices were potential determinants of under-five diarrheal morbidity in the current study area. Thus, health education and promotion should be done with different stakeholders.

\section{Keywords}

\section{Behavioral Factors, Childhood Diarrheal Morbidity, Rural Communities of Ethiopia}

Subject Areas: Epidemiology, Infectious Diseases, Pediatrics, Public Health

\section{Background}

Diarrheal diseases are the second leading cause of child deaths globally and now considered to be the leading cause of child deaths in Africa [1]. Each year, an estimated 2.5 billion cases of diarrhea occur among children under five years of age. More than half of these cases are in Africa and South Asia [2].

In Ethiopia, diarrheal diseases are also important child health problems. According to the recent demographic and health survey of 2011 reported that the national two week-period prevalence of the rural communities was $14 \%$ [3]. Another study conducted in Mecha District, Northern Ethiopia, revealed a two-week prevalence of 18\% [4].

Behavioral (proxy) factors play a primary role in epidemiology of diarrheal diseases. Different studies conducted in Bangladesh, Burma, Philippines, and Papa New Guinea were consistent with an association between a particular hygiene behavior and an increased risk of diarrhea, such behaviors include hand washing before food preparation, open defecation of children, inattention to proper disposal of faeces, the method used by mothers to clean children after defecation, the manner of disposing of the faeces of children and compound hygiene [5]. Several studies in different parts of the world, in daycare centers, and in community settings, have indicated that frequent hand washing, with and without soap (a local substitute like ash, soil, leaves, etc.) results in less diarrheas. Collectively, these studies report a 33 percent reduction in diarrhea from hand washing alone [6]. A review of other available evidence suggests that hand washing with soap could reduce diarrhea incidence by $47 \%$ and save at least one million lives per year [7] [8].

Studies revealed that maternal child care and hygiene practices have important impacts on the occurrence of diarrhea in children [9]. The study conducted in Mecha District, in central Ethiopia, showed children whose mothers with history of diarrheal morbidity were five-fold increase in the odds of getting diarrhea than whose mothers had no history of diarrheal morbidity [4]. Young children are frequently infected with enteric pathogens and their stools are actually an important source of infection for others. Studies have shown that hygienic disposal of children's feces is associated with 30\% - 40\% less risk of diarrhea [10]. Improved feeding practice including breast feeding has positive influence on under-five diarrheal disease. Therefore, mothers should be aware of feeding the baby with a clean spoon, from a cup, or with a special feeding spoon to minimize the risk of bacterial contamination. Bottle-feeding is discouraged at any age. It is usually associated with increased risk of illness, and especially diarrheal disease, because of the difficulty in sterilizing the nipples properly. Bottle-feeding also shortens the period of postpartum amenorrhea and increases the risk of pregnancy [11]. Other findings reported that there is a high chance of contamination and greater risk of diarrhea associated with bottle feeding [12]. The study in peri-urban Peru also showed that 35\% of bottle nipples tested positive for E. coli, an indicator of fecal contamination, and 31\% of teas served in baby bottles were contaminated with E. coli compared with only $2 \%$ of teas served in cups [13]. Determining factors affecting diarrheal morbidity would be helpful in planning and implementation of prevention strategies at the community level. In Ethiopia studies that focus on behavioral determinant of diarrhea in rural setting are sparse. Thus, the objective of this study was investigating behavioral factors on the under-five diarrheal morbidity in the rural communities of Gummer Woreda, Southern Ethiopia.

\section{Methods}

\subsection{Study Area}

The study was conducted in Gummer woreda, Gurage zone, southern Ethiopia. Arekit is the capital town of the 
woreda located at about $65 \mathrm{Km}$ South East of Wolkite town .According to 2007 population census, the total population of Gummer woreda was approximated to 80,163 with the total households of 16,360 [14]. The projected population for 2012 was 92,481 with 18,874 households by considering $2.9 \%$ as natural increase rate of Southern Ethiopia.

\subsubsection{Study Design and Period}

Community based cross-sectional study was conducted from April 30/2012 to June 4/2012.

\subsubsection{Study Participants}

The source population for the study was all households with under-five children in rural community of Gummer woreda. The study population was the sampled households with under-five children among selected kebeles. For households with two or more children under 5 year of age, the index child was selected by a lottery method.

Households with under-five children, mothers/caregivers volunteer to participate in this study and the occurrence of any episode of diarrhea in index child within two weeks prior to survey were included. Mothers/caregivers who were unable to give information of demographic and health characteristics of index child due to health problem, the occurrence of any episode of diarrhea in index child during the survey and mothers/caregivers who did not live at least six-months in the study area were excluded.

\subsection{Sampling}

The sample size was calculated by using the formula for single population proportion, with assumption of $95 \%$ confidence interval $(Z=1.96), d=5 \%$ margin of error, two-week period prevalence of diarrhoea in the rural communities among under-five children $25 \%$ [11]. We went at least two stages down in the sampling process to reach to the final sampling unit. As a result we obliged to multiply our sample size with the design effect of 2 to increase the sample size and minimize the variability. We considered the none-response rate of $10 \%$ in the estimation of the sample size required for the study. The calculated final sample size was 634. Multistage sampling procedure was employed, first by selecting 9 (50\%) kebele from 18 kebeles, using lottery method.

\subsection{Data Collection and Measurements}

Data were collected by using pretested structured questionnaire and observation checklist, which was developed after reviewing relevant literatures and WHO standards.

The questionnaire was first prepared in English, translated into Amharic, and then re-translated back to English to check for its consistency. For data collection process two bachelor degree nurses, one public health officer, two sanitarians, seven public health nurses, and eleven diploma clinical nurses were recruited. Four public health officers were selected as supervisors.

Training for data collectors and supervisors was given for three days before and after pretest on interviewing techniques, purpose of the study and ethical aspects. The principal investigator and supervisors made a day to day on site supervision during the whole period of data collection and checked each questionnaire daily for its completeness and consistency. Pre-test was carried out among five percent of the sampled populations with under-five children in another Kebele with the similar backgrounds as selected kebeles. Based on the result of pre-test, appropriate modifications were made to have the final version.

Regarding with study variables, the two week-period childhood diarrhea prior to the survey was dependent variable. The dependent variables included socioeconomic (family size, age of mother, religion, marital status, education and occupation of mother). Behavioral (proxy) factors included (hand washing practices, presence of feces seen around the pit hole/on the slab, observable faeces in the compound, observable feaces in the neighborhood yard, frequency of feeding/day, breast feeding status, method of feeding supplementary food, time of introducing weaning food, maternal diarrhea, disposal of children feaces).

\subsection{Data Processing and Analysis}

Data were entered and analyzed by using SPSS version 16.0 statistical software. The data were checked for its completeness and consistency during and after entry. Errors identified were corrected after revising the original questionnaires. Descriptive analysis was done using percentages (\%), mean and standard deviation and displayed mainly on tables. 
Bivariate analysis was performed to determine whether there was an association between dependent and independent factors. Then, odds ratio at $95 \%$ confidence interval was used by employing logistic regression model to determine the strength of association between selected variables and for the different risk factor of under-five diarrhea morbidity by controlling for the effect of possible confounders. Further analysis was carried out using stepwise logistic regression analysis by selecting only variables that were statistically significant $(\mathrm{p}<0.05)$ in bivariate analysis. P-value $<0.05$ was considered as a statistically significant in multivariate analysis.

\subsection{Ethical Considerations}

Before the study carried out, research activities were commenced upon approval by Jimma University Ethical and Research Review Committee. The purpose of the study was clearly explained for the study participants as well as consent was obtained from the participating individuals by using the standard method developed for this purpose.

\section{Results}

\subsection{Socio-Demographic Characteristics}

Out of 634 households with at least one under five child, 611 (96.4\%) gave informed consent and were included in the study.

The mean household family size of the participated households was $5.3( \pm 1.5)$ persons. Of 611 households, 360 (58.9\%) had a family size of less than five and 251 (41.1\%) had greater than five. The mean age of mothers who participated in this study was $31.8( \pm 5.2)$ years. Four hundred thirty six mothers/care takers $(71.4 \%)$ were below thirty five years of age while one hundred seventy five mothers/caretakers (28.6\%) were thirty five and above thirty five years of age. Concerning religion of mothers three hundred fourteen (51.4\%) were Muslims. The majority of mothers 598 (97.9) were married, Majority of the respondents 599 (98\%) were Gurage in ethnicity. Five hundred seventy four (93.9\%) mothers were house wives (Table 1).

Table 1. Distribution of respondents by socio-demographic characteristics in the rural community of Gummer Woreda, June 2012.

\begin{tabular}{|c|c|c|c|}
\hline Variables & Response category & Frequency & Percent (\%) \\
\hline \multirow{2}{*}{ Family size $(n=611)$} & $\leq 5$ family & 360 & 58.9 \\
\hline & $>5$ family & 251 & 41.1 \\
\hline \multirow{2}{*}{ Age of Mothers $(n=611)$} & $<35$ years & 436 & 71.4 \\
\hline & $\geq 35$ years & 175 & 28.6 \\
\hline \multirow{3}{*}{ Religion $(n=611)$} & Orthodox & 270 & 44.2 \\
\hline & Protestant & 27 & 4.4 \\
\hline & Muslim & 314 & 51.4 \\
\hline \multirow{4}{*}{ Marital status $(n=611)$} & Married & 598 & 97.9 \\
\hline & Divorced & 5 & 0.8 \\
\hline & Single & 1 & 0.2 \\
\hline & Widowed & 7 & 1.1 \\
\hline \multirow{4}{*}{ Ethnicity (n = 611) } & Gurage & 599 & 98.0 \\
\hline & Silte & 7 & 1.1 \\
\hline & Amahara & 2 & 0.3 \\
\hline & Others $^{*}$ & 3 & 0.5 \\
\hline \multirow{4}{*}{ Maternal education $(n=611)$} & Illiterate & 491 & 80.4 \\
\hline & Read and write & 61 & 10.0 \\
\hline & Primary & 54 & 8.8 \\
\hline & Secondary and above & 5 & 0.8 \\
\hline \multirow{2}{*}{ Maternal Occupation $(n=611)$} & Housewives & 594 & 93.9 \\
\hline & Others $^{* *}$ & 37 & 6.1 \\
\hline
\end{tabular}

Others ${ }^{*}$ : Hadiya, Kambata, Wolayita, Others ${ }^{* *}$ : government employers, private workers. 


\subsection{Feeding Practice}

Of 611 index children, only forty seven (7.7\%) were below 5 months, two hundred fifty one (41.1\%) were 6 - 23 age months while three hundred thirteen (51.2\%) were 24 months and above. The mean age of the index children was about $24.7( \pm 14.09)$ months. Of the total index children, $327(53.5 \%)$ were males and 284 (46.5\%) were females. The mean length of birth space for all births was 30.12 months. The study subjects were interviewed about the history of their childhood diarrhea in the past two-week's period prior to the survey. Accordingly, with in two week-period 89 children exposed to diarrhea and 522 children were not exposed to diarrhea. Regarding current breast feeding status, only 59 (9.7\%) of mothers fed exclusively breast milk (Table 2).

From those mothers who started complementary feeding to their children, two hundred six (37.3\%) started before the child reached 6 months. Concerning the feeding frequency per day for children, out of the total 552 mothers/caretakers who started complementary feeding, nearly half, $282(51.1 \%)$ of mothers/care takers fed their children 3 times per day. As far as the method they used to feed complementary feeding their child, 284 (51.4\%) used their hands and 78 (14.1\%) used bottle to feed. Out of the total 611 mothers/caretakers, only 26 (4.3\%) had diarrhea in the past two weeks of prior to the survey (Table 2).

Concerning hand washing practice of mothers/caretakers, three hundred ninety seven (65\%), claimed to wash their hands before eating. The study also assessed, hand washing practice of mothers/caretakers with soap/ash at each critical moment. Accordingly, very few respondents 109 (17.8\%) were used to wash their hands at least in one and more critical moments. With respect to food/fluid given during diarrheal attack, thirty six (40.4\%) of respondents gave the same amount as usual (Table 2).

Table 2. Feeding practices of children and maternal hygiene practices in the rural communities of Gummer Woreda, June 2012.

\begin{tabular}{|c|c|c|c|}
\hline Variables & Response category & Frequency & Percent (\%) \\
\hline \multirow{3}{*}{ Current breast feeding status of the child $(n=611)$} & Exclusive breast feeding & 59 & 9.7 \\
\hline & Partial breast feeding & 289 & 47.3 \\
\hline & Not breast feeding & 263 & 43.0 \\
\hline \multirow{2}{*}{ Starting age of supplementary feeding $(n=552)$} & $<6$ months & 206 & 37.3 \\
\hline & $\geq 6$ months & 346 & 62.7 \\
\hline \multirow{3}{*}{ Frequency of feeding/day $(n=552)$} & $<3$ times & 74 & 13.4 \\
\hline & 3 times & 282 & 51.1 \\
\hline & $>3$ times & 196 & 35.5 \\
\hline \multirow{4}{*}{ Methods used in complementary feeding $(n=552)$} & Hand feeding & 284 & 51.4 \\
\hline & Cup and spoon & 256 & 46.4 \\
\hline & Bottle feeding & 78 & 14.1 \\
\hline & Others $^{*}$ & 33 & 5.9 \\
\hline \multirow{2}{*}{ History of maternal diarrhea $(n=611)$} & Yes & 26 & 4.3 \\
\hline & No & 585 & 95.7 \\
\hline \multirow{6}{*}{ Time of hand washing of mothers $(n=611)$} & Before eating & 397 & 65 \\
\hline & Before preparing food & 220 & 36 \\
\hline & Before feeding child & 142 & 23.2 \\
\hline & After visiting latrine & 175 & 29 \\
\hline & After cleansing child’s bottom & 102 & 16.7 \\
\hline & At all critical moments & 19 & 3.2 \\
\hline \multirow{2}{*}{ Hand washing practice with $(n=611)$} & Only water & 502 & 82.2 \\
\hline & Soap/ash \& water & 109 & 17.8 \\
\hline \multirow{3}{*}{ Fluids/foods Provided during diarrhea $(n=89)$} & The same amount & 36 & 40.4 \\
\hline & Less amount & 33 & 37.1 \\
\hline & More amount & 20 & 22.5 \\
\hline
\end{tabular}

Others ${ }^{*}$ : Mainly includes children who ate by themselves, i.e. without the help of mothers/caretakers. 


\subsection{Hygienic Behavior}

Out of 611 households, 166 (27.5\%) of households were observed that there were faeces around the pit hole/on slab of the latrine. Respondents were asked about children's faeces disposal practices, the majority 385 (63.8\%) disposed properly while $36.2 \%$ of respondents disposed improperly. During the survey $16 \%$ of households' feaces were observed around the compound/yard and only 5.2\% of households' feaces were observed in the neighborhood yard.

\subsection{Factors Associated with Under-Five Diarrheal Morbidity}

Bivariate and multivariate logistic regressions were used to identify the characteristics that differentiated children who had and did not have diarrhea during the two weeks period. The dichotomous variable was defined as one child who had diarrhea and zero the child who did not have diarrhea during the two week-period before the survey. Coefficients were expressed as crude and adjusted OR in relation to the referent category.

Variables significantly associated in bivariate analysis were current breast feeding ,hand washing after visiting the latrine, maternal hand washing practice with/out soap or ash, history of maternal diarrhea and observable faeces seen in neighborhood yard $(\mathrm{p}<0.05)$ and bottle feeding and presence of faeces seen around the pit hole/on the slab $(\mathrm{p}<0.001)$ (Table 3$)$.

The crude OR shows significant differences of the control variables on the risk of childhood diarrhea. Though, the analysis doesn't assess the independent effects of the covariates at this level. To identify the independent effects, the multivariate logistic regression was used. Variables which were found to be associated significantly in a bivariate analysis were re-examined independently using multivariate logistic regression analysis by controlling other possible confounders.

Variables such as hand washing after visiting the latrine, practice of bottle feeding, presence of faeces seen around the pit hole/on the slab, and history of maternal diarrhea were remained independent predictors of under-five diarrheal morbidity prevalence.

Respondents who used bottle feeding method to feed their children were 3 times more likely to had diarrheal morbidity as compared to respondents who didn't use bottle feeding method (AOR: 2.866, 95\%CI: (1.588, 5.170). On the other hand, practice of hand washing after visiting latrine is associated with the occurrence of childhood diarrhea. Households who practiced washing their hands with soap/ash prevent childhood diarrhea two times more likely than households who practiced without soap/ash (AOR: 2.080, 95\%CI: (1.076, 4.021). The presence of observable faeces around the pit hole/slab in their latrine of the households also had statistically significant association with the occurrence of childhood diarrheal morbidity. The risk of the occurrence of childhood diarrheal morbidity was 2.4 times higher in the households with the presence of observable faeces around the pit hole/slab than households with no observable faeces around the pit hole/slab of their latrine (AOR: 2.391, 95\% CI: (1.444, 3.959). Children from those mothers who had history of diarrhea were three times more vulnerable to diarrhea than children from those mothers who didn't not have diarrhea (AOR: 3.158, 95\%CI: (1.240, 8.045) (Table 3).

\section{Discussion}

This study assessed behavioral factors associated with under-five children diarrheal morbidity in rural community of Gummer Woreda.

The study revealed that childhood diarrheal morbidity prevalence rate in two weeks-period was $14.6 \%$ in rural community of Gummer Woreda. This study is consistent with the recent report of Ethiopia Demographic and Health Survey, which showed the two week-period prevalence of diarrheal morbidity $14 \%$ [3]. However, the current study is inconsistent with study of Mecha District, Northern Ethiopia, revealed a two-week prevalence of $18 \%$ [4]. This failure to match might be variation in socio-economic and demographic, environmental and behavioral characteristics of study households.

In current study, presence or absence of faeces around the pit-hole/on the slab was predictor of under-five children diarrheal morbidity. The odds of developing childhood diarrhea was 2.4 times higher in children from those households in which feaces were observed around the pit hole or on the slab compared with households in which feaces were not observed around the pit hole or on the slab. On the other hand, hand washing practice after visiting latrine showed statistically significant association with childhood diarrhea. Households who practiced washing their hands with soap/ash prevent childhood diarrhea two times (AOR: 2.080, 95\%CI (1.076, 4.021) 
Table 3. Factors associated with under-five childhood diarrheal morbidity, in rural community of Gummer Woreda, June 2012.

\begin{tabular}{|c|c|c|c|c|}
\hline \multirow{2}{*}{ Characteristics } & \multicolumn{2}{|c|}{ Diarrhea } & \multirow{2}{*}{ COR at $95 \% \mathrm{CI}$} & \multirow{2}{*}{ AOR at $95 \% \mathrm{CI}$} \\
\hline & Yes & No & & \\
\hline \multicolumn{5}{|l|}{ Age of mother/caretaker } \\
\hline$<35$ years & $50(11.5)$ & $386(88.5)$ & $0.452(0.285,0.717)$ & $0.415(0.246,0.702)$ \\
\hline$\geq 35$ years & $39(22.3)$ & $136(77.7)$ & 1.00 & 1.00 \\
\hline \multicolumn{5}{|l|}{ Maternal occupation } \\
\hline Housewife & 79 (13.8) & $495(86.2)$ & 1.00 & 1.00 \\
\hline Others & $10(27)$ & $27(73)$ & $2.321(1.082,4.979)$ & $3.085(1.299,7.325)$ \\
\hline \multicolumn{5}{|l|}{ Type of water source } \\
\hline Protected & $43(12.1)$ & $311(87.9)$ & 1.00 & 1.00 \\
\hline Unprotected & 46 (17.9) & $211(82.1)$ & $1.577(1.004,2.475)$ & $1.502(0.994,2.401)$ \\
\hline \multicolumn{5}{|c|}{ Distance to water source(round) } \\
\hline$<30$ minutes & $20(8.6)$ & $212(91.4)$ & 1.00 & 1.00 \\
\hline$\geq 30$ minutes & 69 (18.2) & $310(81.8)$ & 2.359 (1.392, 3.998) & $2.157(1.214,3.834)$ \\
\hline \multicolumn{5}{|c|}{ Hand washing facilities around the } \\
\hline Yes & $20(9.6)$ & $188(90.4)$ & 1.00 & 1.00 \\
\hline No & $67(17)$ & $328(83)$ & $1.920(1.129,3.264)$ & $1.851(0.918,3.213)$ \\
\hline \multicolumn{5}{|l|}{ Current breast feeding } \\
\hline Exclusive breast feeding & $4(6.8)$ & $55(93.2)$ & 1.00 & 1.00 \\
\hline Partially breast feeding & $53(18.3)$ & $236(81.7)$ & $3.088(1.072,8.893)$ & $2.986(0.913,8.614)$ \\
\hline Not breast feeding & $32(12.2)$ & $231(87.8)$ & $1.905(0.647,5.610)$ & $1.842(0.791,5.317)$ \\
\hline \multicolumn{5}{|l|}{ Bottle feeding } \\
\hline Yes & $24(30.8)$ & $54(69.2)$ & $3.009(1.735,5.220)$ & $2.757(1.494,5.087)$ \\
\hline No & $61(12.9)$ & $413(87.1)$ & 1.00 & 1.00 \\
\hline \multicolumn{5}{|c|}{ Hand washing after visiting latrine } \\
\hline Yes & $16(9.1)$ & $159(90.9)$ & 1.00 & 1.00 \\
\hline No & $71(16.6)$ & $357(83.4)$ & $1.976(1.114,3.508)$ & $1.886(0.984,3.332)$ \\
\hline \multicolumn{5}{|l|}{ Hand washing practice with } \\
\hline Water only & $81(16.1)$ & $421(83.9)$ & $2.429(1.138,5.184)$ & $2.210(0.845,4.862)$ \\
\hline Soap/ash water & $8(7.3)$ & $101(92.7)$ & 1.00 & 1.00 \\
\hline \multicolumn{5}{|c|}{ Presence of feaces around the pit hole } \\
\hline Yes & $40(24.1)$ & $126(75.9)$ & $2.634(1.651,4.202)$ & $2.253(1.344,3.777)$ \\
\hline No & 47 (10.8) & $390(89.2)$ & 1.00 & 1.00 \\
\hline
\end{tabular}

more likely than households who practiced without soap/ash. The study is supported by several studies in different parts of the world, in daycare centers, and in community settings, which have indicated that frequent hand washing, with and without soap (a local substitute like ash, soil, leaves, etc.) results in fewer diarrheas. Collectively, these studies report a 33 percent reduction in diarrhea from hand washing alone [6]. A review of other available evidence also suggests that hand washing with soap could reduce diarrhea incidence by $47 \%$ and save at least one million lives per year [7] [8].

Children from households who used bottle feeding method had almost 3 times more likely to have diarrhea than households who didn't use bottle feeding method [AOR: 2.866, 95\%CI $(1.588,5.170)]$. This might be due to use of unclean bottle as well as bottles are particularly important route of transmission of pathogens. The study is also supported by the study in peri-urban Peru which showed that $35 \%$ of bottle nipples tested positive for E. coli, an indicator of fecal contamination [13]. 
Regarding to maternal history of diarrheal morbidity, children from whose mothers had history of diarrhea were three times [AOR: 3.158, 95\%CI $(1.240,8.045)]$ more vulnerable than children from whose mothers didn't not have diarrheal morbidity. This might be due to mothers are food handlers of the family, and also they are usual child care providers. The study is in line with the study conducted in Mecha District, in central Ethiopia, showed children whose mothers that history of diarrheal morbidity was five-fold increase in the odds of getting diarrhea than whose mothers had no history of diarrheal morbidity [4].

In present study hand washing before preparing food, cleansing of child's bottom, child's feaces disposal manner and observable feaces in the compound had no association with childhood diarrhea,. In contrast, various studies conducted in Bangladesh, Burma, Philippines, and Papa New Guinea were consistent with an association between a particular hygiene behavior and an increased risk of diarrhea, such behaviors include hand washing before food preparation, open defecation of children, inattention to proper disposal of faeces, the method used by mothers to clean children after defecation, the manner of disposing of the faeces of children and compound hygiene [5].

\section{Limitation of the Study}

Recall bias by mothers/caregivers during interview of two weeks occurrence of childhood diarrhea was very likely. As it is cross-sectional, it fails to show seasonal variability in the occurrence of diarrheal morbidity

\section{Conclusions}

The findings of this study showed that the two weeks period prevalence of under-five diarrheal morbidity was $14.6 \%$. Hand washing after visiting the latrine, practice of bottle feeding, presence of faeces seen around the pit hole/on the slab, and history of maternal diarrhea were the major factors affecting under five diarrheal morbidity.

Health education should be given by health extension workers, sanitarians, public nurses and other nongovernmental organizations on water, sanitation and hygiene activities to communities. For better intervention, this study recommends further study that focus on rural community with possible factors that are responsible for high prevalence of childhood diarrhea, water quality and climatic factors.

\section{Acknowledgements}

We would like to thank Gummer Woreda administration and health office for their financial and material support to conduct this study. We are also very glad to express our appreciation to data collectors, supervisors and study participants.

\section{Competing Interests}

The authors declare that they have no competing interests.

\section{Authors' Contribution}

Eyasu Gambura wrote the proposal, participated in data collection, analyzed the data and drafted the paper, Wondwossen Birke and Gudina Terefe, approved the proposal, participated in data analysis and revised subsequent draft of the paper, Tariku Laelago and Tadele Yohanes extensively participated in data analysis. All authors read and approved the final manuscript.

\section{References}

[1] Water, Sanitation and Hygiene Portfolio Review. Department for International Development, March 2012, 28.

[2] Boschi Pinto, C., et al. (2009) The Global Burden of Childhood Diarrhoea. In: Ehiri, J.E. and Meremikwu, M., Eds., International Maternal and Child Health, WHO, Geneva, 225-243. http://dx.doi.org/10.1007/b106524_13

[3] Ethiopia Demographic and Health Survey (EDHS) (2011) Central Statistics Authority: Addis Ababa Ethiopia, ICF International, Calverton.

[4] Muluken, D. (2009) Does the Effect of Water and Sanitation Vary by Maternal Education on Childhood Diarrhea among Under-Five Children in Mecha District West Gojam Ethiopia? AAU, Adis Ababa. 
[5] Baltazar, J.C., et al. (1997) Hygiene Behaviour and Hospitalized Severe Childhood Diarrhea: A Case-Control Study. Bulletin of WHO, 71, 323-327.

[6] Huttly, S.R.A., et al. (1997) Prevention of Diarrhea in Young Children in Developing Countries. Bulletin of WHO, 75, 163-174.

[7] World Water Day (WWD) (2001) Water, Sanitation and Hygiene at a Glance. http://www.cmhealth.org/

[8] World Bank (2002) Water Supply and Sanitation. Hygiene and Sanitation Promotion: Why Promote Sanitation.

[9] Woldemicael, G. (2001) Diarrheal Morbidity among Young Children in Eritrea: Environmental and Socio-Economic Determinants. Journal of Health, Population and Nutrition, 19, 83-90.

[10] World Health Organization (WHO) (2000) African Regional Water Supply and Sanitation Assessment.

[11] Ethiopia Demographic and Health Survey (DHS) (2005) Central Statistics Authority \& ORC Marco. Addis Ababa, Ethiopia and Calverton, Maryland, 140-150.

[12] Getaneh, T., et al. (1997) Diarrhea Morbidity in Urban Area of South-West Ethiopia. East African Medical Journal, 74, 491-494.

[13] Black, R.E., et al. (1989) Incidence and Etiology of Infantile Diarrhea and Major Routes of Transmission in Huascar. American Journal of Epidemiology, 129, 785-99.

[14] Federal Democratic Republic of Ethiopia Population Census commission (FDRECC) (2008) Summary and Statistical Report of the 2007 Population and Housing Census, December 2008, 76-83.

\section{Abbreviations and Acronym}

AOR: Adjusted odd ratio;

CI: Confidence interval;

COR: Crude odds ratio;

SD: Standard deviation;

WHO: World Health Organization 